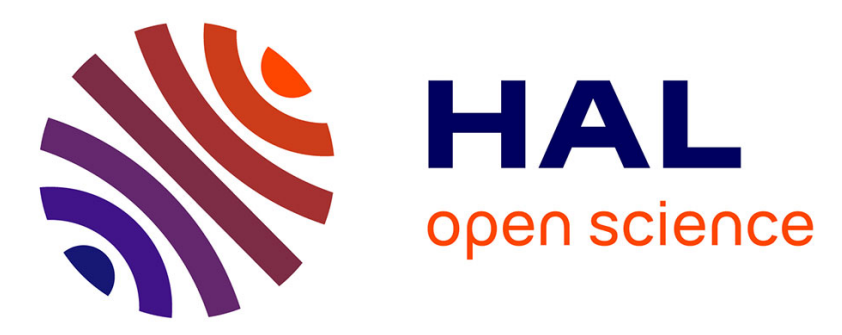

\title{
Capacitively coupled electrical substitution for resistive bolometer enhancement
}

\author{
Matthieu Denoual, Sébastien Delaunay, Gilles Allègre, Didier Robbes
}

\section{To cite this version:}

Matthieu Denoual, Sébastien Delaunay, Gilles Allègre, Didier Robbes. Capacitively coupled electrical substitution for resistive bolometer enhancement. Measurement Science and Technology, 2009, pp.015105. hal-00980248

\section{HAL Id: hal-00980248 \\ https://hal.science/hal-00980248}

Submitted on 22 Apr 2014

HAL is a multi-disciplinary open access archive for the deposit and dissemination of scientific research documents, whether they are published or not. The documents may come from teaching and research institutions in France or abroad, or from public or private research centers.
L'archive ouverte pluridisciplinaire HAL, est destinée au dépôt et à la diffusion de documents scientifiques de niveau recherche, publiés ou non, émanant des établissements d'enseignement et de recherche français ou étrangers, des laboratoires publics ou privés. 


\title{
Capacitively coupled electrical substitution for resistive bolometer enhancement
}

\author{
M Denoual ${ }^{1}$, S Delaunay ${ }^{1}$, G Allègre ${ }^{1}$ and D Robbes $^{1,2}$ \\ ${ }^{1}$ GREYC-ENSICAEN, université de basse Normandie, 6 bd maréchal Juin, 14000 Caen, France \\ ${ }^{2}$ INPHYNIX, bd Henri Becquerel, 14076 Caen, Cedex 05, France \\ E-mail:mdenoual@greyc.ensicaen.fr
}

\begin{abstract}
A new electrical substitution method for resistive bolometers is proposed to operate them in a closed-loop configuration. This method was implemented and evaluated with a resistive bolometer based on metallic layers over a $120 \mu \mathrm{m}$ thick glass membrane. Based on an electrical substitution (ES) directly at the place of the resistive sensing element, the new method allows for space savings and a simplification of the technological manufacturing while maintaining the time response improvement linked to a closed-loop operation. Compared to a previously available ES solution, this new method is applicable to all resistive bolometers. Time response and signal-to-noise ratio (SNR) were evaluated through measurements and compared for three operation configurations of the resistive bolometer: open loop, classical ES closed loop and with the proposed capacitively coupled electrical substitution (CCES) closed loop.
\end{abstract}

Keywords: bolometer, electrical substitution, thermal feedback

\section{Introduction}

Recently, demand for a cheap and uncooled infrared (IR) detector has grown both for civilian and military applications [1]. The automobile industry's interest in those nocturnal vision systems for safety and assisted driving corresponds to high market volumes in the coming years. For uncooled applications, the thermal detector has an advantage over the photon detector, especially concerning noise [2]. Thermal detectors can be divided into three types: bolometer, thermopile and pyroelectric detector. The first one has advantages as far as manufacturing and simple use are concerned. Indeed, it does not require any added reference junction nor a chopper. Hence pushed integration can be obtained compared to a thermopile resulting in microbolometers [3] which are then a simple and cheap solution for IR detection.

A bolometer converts absorbed IR radiation into heat, which in turn changes the resistance of the absorption layer in the case of a resistive bolometer or the pressure of a gas cell in the Golay cell case. A bolometer can be modeled as an IR-sensitive element of thermal mass $C_{\text {th }}$ linked via a thermal conductance $G_{\text {th }}$ to a substrate serving as the heat sink or thermostat (figure 1). The performance of the bolometer is characterized by certain figures of merit such as the temperature coefficient of resistance (TCR or $\alpha$ ) of the temperature sensing resistor, its responsivity $(R)$, its specific detectivity $\left(D^{*}\right)$ and its intrinsic time constant $\tau_{\text {th }}=$ $C_{\text {th }} / G_{\text {th }}$ [4]. The specific detectivity is derived from the sensor's responsivity, its geometrical dimensions and its noise equivalent power. Responsivity is therefore a key criterion for bolometer comparison, it is expressed as follows:

$$
R[\mathrm{~V} / \mathrm{W}]=\frac{V_{s}}{P_{\mathrm{IR} \mathrm{rad}}}=\frac{\alpha \eta I_{\mathrm{bias}} R_{\mathrm{sensing}}}{\left(G_{\mathrm{th}}^{2}+\omega^{2} C_{\mathrm{th}}^{2}\right)^{1 / 2}},
$$

where $R_{\text {sensing }}$ is the bolometer resistance (sensing resistor), $I_{\text {bias }}$ is the bias current, $\eta$ is the absorption coefficient of the 


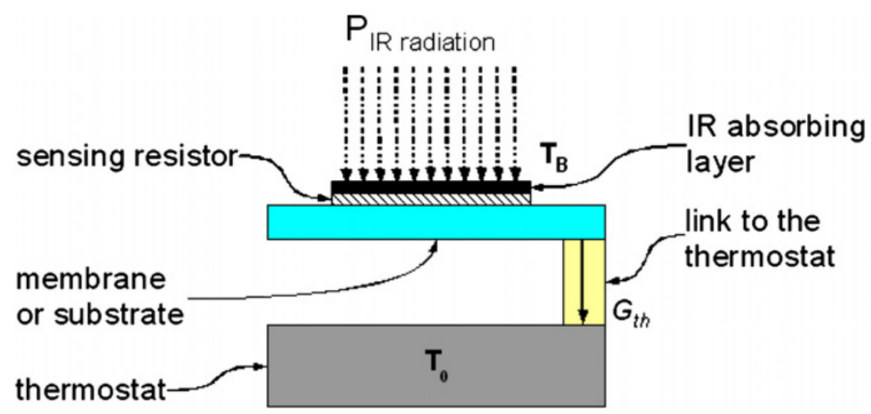

Figure 1. Sketch of a resistive bolometer. The incident IR power is absorbed and converted into heat. The heat raises the temperature of the sensing element, part of a thermal system coupled to a thermostat at a constant temperature. The resistance change of the resistive sensing element is measured.

absorption layer of the device and $P_{\mathrm{IR} \text { rad }}$ is the input radiative power. The thermal mass should be reduced to optimize the time constant, whereas thermal conductance choice would result from a compromise between responsivity (requires a small $G_{\mathrm{th}}$ ) and time constant (requires a high $G_{\mathrm{th}}$ ).

Performances of bolometers can be improved through the design and manufacturing of the sensor or through its implementation and use. Considering the design and manufacturing of the sensor, the research works focus on using high TCR materials, for example YBCO or LSMO $[5,6]$, to increase sensor responsivity or miniaturization or manufacturing over membranes [7-9] to reduce thermal mass and optimize the thermal conductance under the responsivity/time constant compromise.

Considering the implementation and use, for a given sensor, substantial improvements in linearity, dynamic range and time constant can be obtained through a closed-loop configuration as well as for any physical system. Linearity is a crucial issue for resistive bolometer especially when superconducting materials [10] or functional oxides [11] are used because of non-linear resistance versus temperature characteristics. Heat feedback can ensure that the sensing resistor temperature is kept constant at a working temperature. Heat, the physical feedback quantity, can be produced thanks to optical means, an IR feedback source, or thanks to the Joule effect with resistors placed close to the sensing resistor in the electrical substitution case (ES). Whatever the heat production means, the feedback power will exactly compensate incoming radiative power [12-14] so as to ensure a constant of the total heat dissipated in the sensing resistor location:

$$
P_{\text {Total }}=\eta \cdot P_{\mathrm{IR} \text { radiation }}+P_{\text {Heat Feedback }}+P_{\text {Bias }} .
$$

$P_{\text {Bias }}$ corresponds to the power dissipated in the sensing resistor of the bolometer due to the biasing current $I_{\text {bias }}$.

Considering ES, in the classical configuration, heat power (3) is produced through the Joule effect in an additional resistor in the proximity of the sensing resistor (figure 2(a)), next to the sensing element [12] or on the backside when a membrane is used [14]:

$$
P_{\text {Heat Feedback }_{E S}}=\frac{\beta \cdot V_{\text {Heat Feedback }}^{2}}{R_{\text {Heating }}},
$$

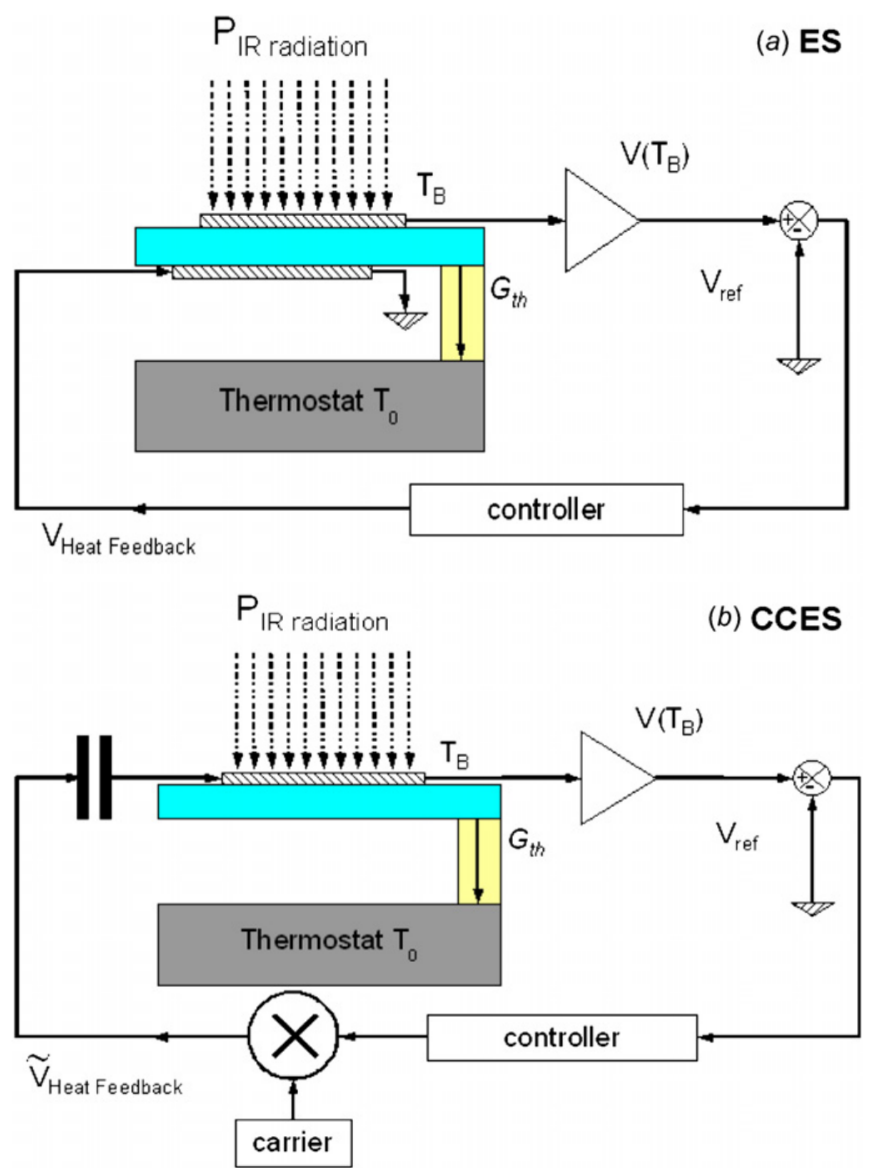

Figure 2. Electrical substitution configurations. (a) The classical ES configuration using an additional resistor for feedback heating at the bottom side of the membrane. (b) The proposed capacitive coupled CCES, using the sensing resistor for the feedback. Carrier frequency is typically between 100 and $10 \mathrm{MHz} . T_{B}$ is the temperature of the sensing resistor, $V\left(T_{B}\right)$ is the readout electronics output voltage, $V_{\text {ref }}$ is the reference voltage that imposes a closed-loop operating point, $I_{\text {Heat Feedback }}$ is the current that feeds back the power into the system by flowing into the heating resistor, ES case or into the sensing resistor, CCES case and $G_{\text {th }}$ is the thermal conductance of the device.

the factor $\beta(<1)$ refers to the fact that only a fraction of the Joule heat dissipated by the heating resistor goes to the sensing resistor.

The closed-loop configuration can also improve measurement accuracy thanks to the optimization of the placement of the sensing resistor bias point. This is of great importance when transition-edge materials are considered for temperature sensing in order to place them at their highest TCR value. This is classically operated for extra low temperature cooled bolometers [13]. The electrical substitution (ES) feedback concept is different from electrothermal feedback (ETF) described in $[15,16]$ for superconducting bolometers, based on the fact that when a superconductive film is voltage biased and the substrate is cooled far below the transition temperature, the film can be made to self-regulate temperature within its transition. As the film cools, its resistance drops toward zero, and the Joule heating increases. A stable equilibrium is established when the Joule heating matches the heat loss in the substrate. The feedback signal is the change 
in the Joule power in the film, the product of the bias voltage and the measured current. Potentially those two approaches, ETF and ES, could be combined in the case of superconductive transition-edge bolometers.

Pre-existing optical or electrical feedback means both require an additional power source. In this work, we propose a new feedback configuration that does not require an extra optical IR source or any heating resistor; therefore it can be applied to every existing bolometer without any material change. Only the readout and feedback controller electronics are adapted. The principle is to apply the feedback heat directly to the sensing resistive element.

In this paper, first a novel electrical substitution configuration, the capacitively coupled electrical substitution (CCES), is described. Then its implementation with a glass substrate bolometer is presented. The bolometer is described and characterized. The performances of the CCES configuration are measured and compared to open-loop and classical ES closed-loop configurations. Time response and SNR validate the proper functioning of the proposed method.

\section{Principle of the capacitively coupled electrical substitution}

As mentioned in the introduction, the classical ES requires extra heating resistor to feedback heat close to the sensing resistor leading to the use of two resistors. A sketch of the principle of the conventional ES configuration is presented in figure 2(a). A sketch of the principle of the capacitively coupled configuration is presented in figure $2(b)$. In that case, only one resistor is used, the heat feedback is directly produced by the sensing resistor. But a dc feedback controlled current cannot directly flow into the sensing resistor because then the electrical bias point would be changed. The solution is to modulate the feedback power and couple it to the sensing resistor, making sure that the spectral domain of the feedback signal is over the bandwidth of the open-loop system (mainly limited by the thermal cutoff frequency of the bolometer). The feedback voltage applied to the sensing resistor is much larger in amplitude ( $\mathrm{mV}$ range) than the voltage variation due to temperature to be measured ( $\mu \mathrm{V}$ range), hence they have to be spectrally separated to allow easy filtering of the measured signal. Therefore the power feedback is modulated at a typical frequency of several hundreds of $\mathrm{kHz}$. Then the bias point of the sensing resistor is not altered and the RMS coupled power ensures the feedback operation, and therefore the stability of the temperature bias point $T_{\text {working }}$ while keeping the mean electrical bias current $I_{\text {bias }}^{-}$point unchanged.

Then the total dissipated power in the sensing resistor is

$$
\begin{aligned}
& \overline{P_{\text {Joule Heat }}}=P_{\text {Bias }}+P_{\text {Heat Feedback }} \text { CCES } \\
& \overline{P_{\text {Joule Heat }}}=R_{\text {sensing }}+I_{\text {bias }}^{2}+R_{\text {sensing }} \cdot I_{\text {Heat Feedback }}^{2},
\end{aligned}
$$

where $I_{\text {Heat Feedback }}$ is the mean square current feedback.

The readout electronics have to be completed with electronic filters suppressing the modulation frequency of the feedback power at the input of the first amplification stage to prevent its saturation and to filter this frequency from the output measurement signal.

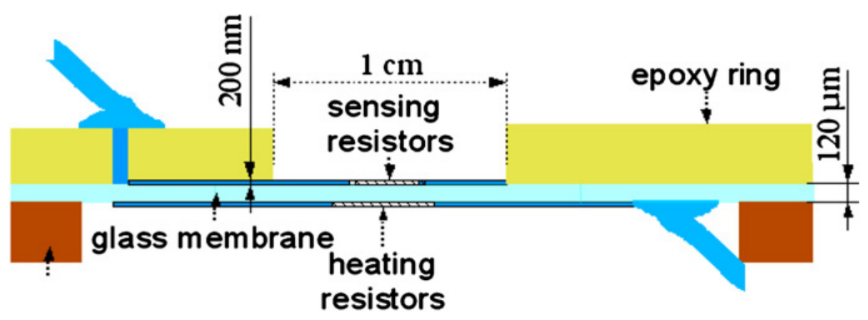

Figure 3. Sketch of the glass bolometer structure with a sensing resistor on the upper side and a heating resistor at the bottom side for characterization.

\section{Experiments}

\subsection{Description of the device}

The bolometer chosen for the evaluation of the proposed method needed to involve heating resistors to be compared with the classical ES configuration. To prevent it from destruction during feedback experiments, a robust and simple glass membrane device made of a pattern mask already available was used [14]. This bolometer consists of a $120 \mu \mathrm{m}$ thick glass membrane. Metallic chromium and gold layers, of $20 \mathrm{~nm}$ and $200 \mathrm{~nm}$, respectively, were deposited on both sides and patterned to form the resistors. Upper side resistors, seeing the incident radiation, are the sensing resistors. Only one is used during the experiments. The bottom side resistors, used for heat feedback or heat stimulation are the heating resistors $R_{\text {heating1 }}$ and $R_{\text {heating2 }}$, respectively (figures 3 and 4). Then the membrane was assembled on a copper holder for heat conduction to the thermostat and for ease of handling.

\subsection{Experimental setup}

For the experiments, the device was placed in a vacuum chamber connected to an Alcatel primary pump. A Pirani gauge was used for monitoring the pressure during the measures. All measures were carried out under 20 mTorr vacuum pressure and at room temperature. A standard lab power source was used to supply the signal conditioning and control feedback electronic boards. Two Philips PM 5131 function generators were used for input power stimulation and HF carrier production in the case of the CCES. The modulation of the feedback voltage was then done with an analog AD633 multiplier. For measurement purpose, the output signal passed through a $50 \mathrm{~Hz}$ passive double-tee cutoff filter to reject power grid noise. The signal was observed using a Tektronic TDS 3064B oscilloscope, and measures were made using an HP 34401 A multimeter. For spectral analysis an HP 3562 A dynamic signal analyzer was used. A sketch of the experimental setup is shown in figure 5 .

During all the experiments, the bolometer was current biased with $1 \mathrm{~mA}$. Differential measurement was performed using a dummy resistor kept at room temperature and distant from the bolometer. Filters used at the input of the amplification stage had a $3 \mathrm{~Hz}$ cut-off frequency.

Power stimulation was done thanks to the heat power produced by a heater resistor at the bottom of the device 


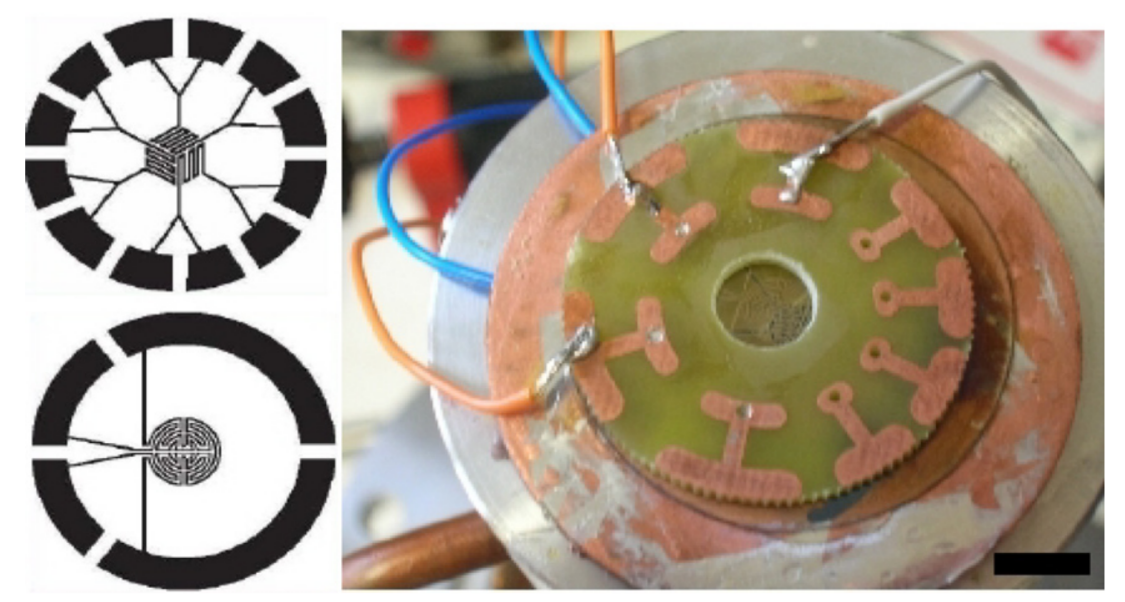

Figure 4. Left: drawing of the mask of the three sensing resistors (top) and the two heating resistors (bottom). All resistor designs are placed within a $0.5 \mathrm{~cm}$ diameter circle. Right: picture of the device wire-bonded and packaged. The hole at the center of the epoxy ring shows the sensing resistor and heater (scale bar is $1 \mathrm{~cm}$ ).

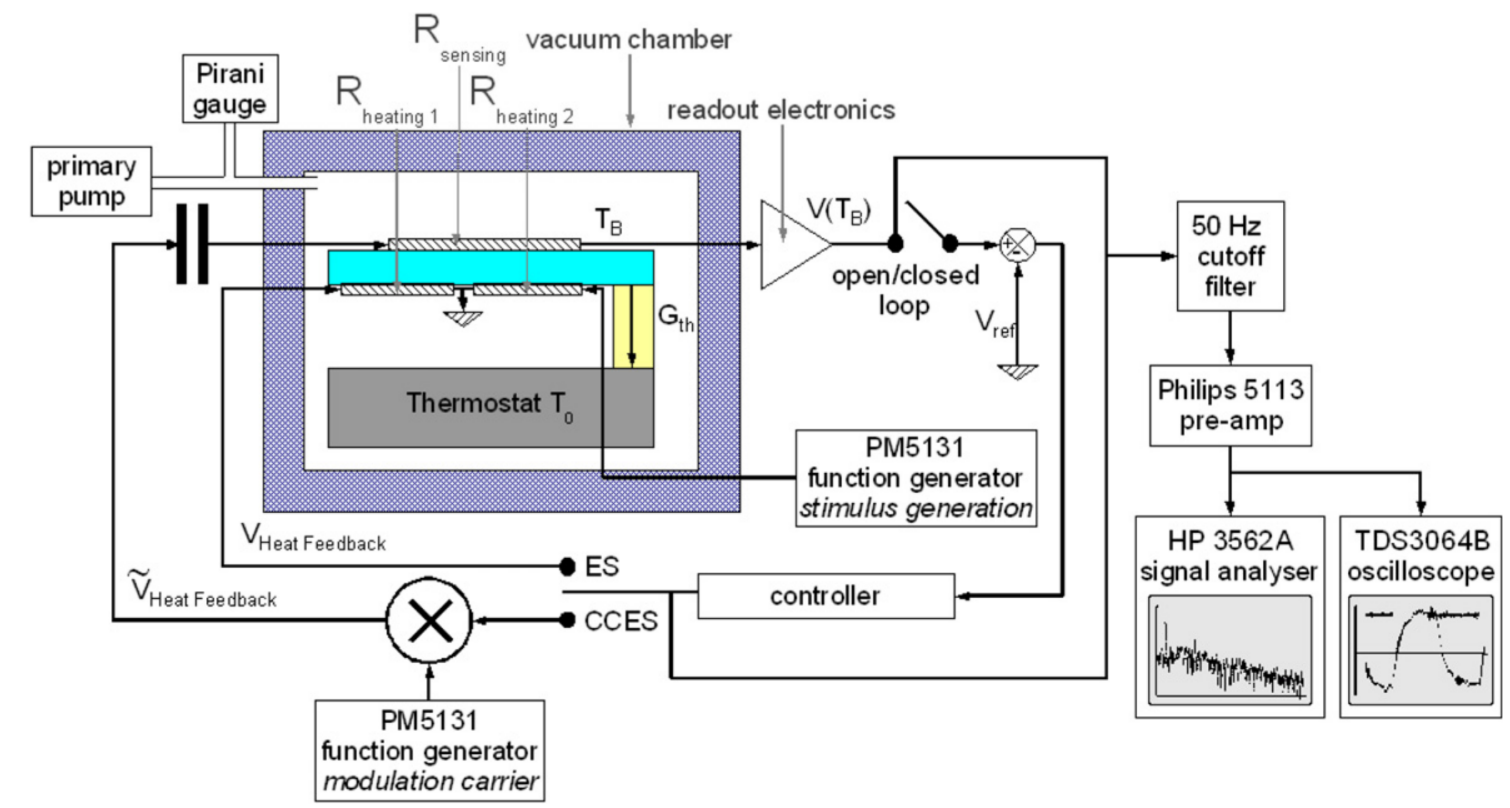

Figure 5. Sketch of the experimental setup with the bolometer device in a vacuum chamber. Three resistors are used: a sensing resistor for temperature measurement, a heating resistor for the power stimulation and another heating resistor for the classical ES configuration when used. Output signals are analyzed both for time and frequency.

$\left(R_{\text {heating2 }}\right)$. This solution allows for the calibration of the input stimulation thanks to voltage control. This allows us to get rid of absorption coefficient identification of the absorption layer on the top of the microbolometer and to carry out simple experiments without any optics.

\subsection{Device characterization}

The resistance of the sensing and heater resistors were measured to be $R_{\text {sensing }}=80.6 \Omega, R_{\text {heating } 1}=4.5 \mathrm{k} \Omega$ and $R_{\text {heating2 }}=11.1 \mathrm{k} \Omega$.

The device was placed in an oven to evaluate the TCR of the metallic sputtered resistors. A TCR of $9.2 \times 10^{-4} \mathrm{~K}^{-1}$ was measured. This quite low value is imputed to the deposition quality.
The thermal properties of the device were derived from step-response experiments performed in an open-loop configuration. Power steps of $1 \mathrm{~mW}$ were applied to the backside heating resistor of the device, the thermal conductance was calculated from the temperature measured by the sensing resistor and the responsivity assuming no heat power leakage. $G_{\text {th }}=1.25 \mathrm{~mW} \mathrm{~K}^{-1}$ was obtained; it mainly corresponds to the glass membrane thermal conductance. The thermal mass $C_{\mathrm{th}}=14.6 \mathrm{~mJ} \mathrm{~K}^{-1}$ was deduced from the measured time of $\tau_{\text {th }}=11.6 \mathrm{~s}$.

These results are in accordance with theory that leads to $1.55 \mathrm{~mW} \mathrm{~K}^{-1}$ if we consider the thermal capacitance of the $1 \mathrm{~cm}$ diameter volume at the center of the device and a specific heat of $750 \mathrm{~J} \mathrm{~kg}^{-1} \mathrm{~K}^{-1}$ for glass, and leads to a thermal conductance of $1.47 \mathrm{~mW} \mathrm{~K}^{-1}$ considering a glass thermal 


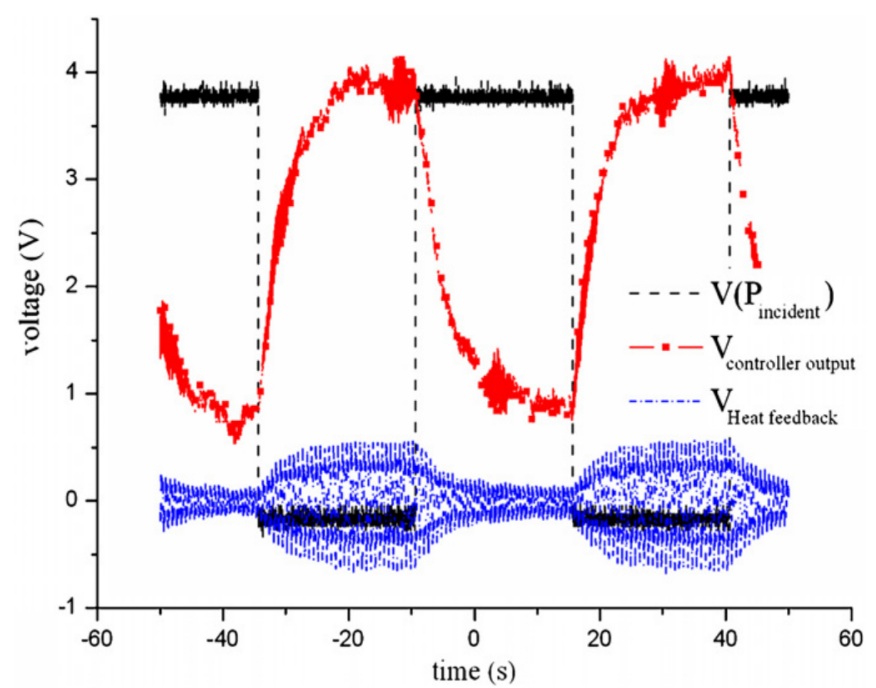

Figure 6. CCES closed-loop signals. Input stimulation, $V\left(P_{\text {incident }}\right)$, is $1.38 \mathrm{~mW}(3.9 \mathrm{~V}$ in the $11.1 \mathrm{k} \Omega$ heating resistor). The feedback is direct with unity controller gain. Fitted time constant response is $4 \mathrm{~s}$. Carrier frequency is $200 \mathrm{kHz}$. The feedback voltage recorded here corresponds to a power of $1.52 \mathrm{~mW}(0.35 \mathrm{~V}$ in the $80.6 \Omega$ sensing resistor).

conductivity $\lambda$ of $1.35 \mathrm{~W} \mathrm{~m}^{-1} \mathrm{~K}^{-1}$, the sensing resistor radius $r_{1}=0.25 \mathrm{~cm}$, the membrane radius $r_{2}=0.5 \mathrm{~cm}$ and $G_{\mathrm{th}}$ expressed as $G_{\text {th }}=2 \pi \cdot \lambda \cdot t / \ln \left(r_{2} / r_{1}\right)$ [3, 17]. These rather poor thermal characteristics are the drawback of the device robustness and simplicity.

\section{Results}

First, the CCES was implemented and tested then a comparative survey was carried out to compare open-loop, $\mathrm{ES}$ and CCES configurations in terms of time response and signal-to-noise ratio.

\subsection{CCES implementation}

Before closing the system with the heat feedback, the working point has to be defined. After thermal stabilization in the vacuum chamber of the device, a modulated power is applied to the sensing resistor using the working reference voltage input and in open loop to set the working temperature. It was typically set 1 to $3 \mathrm{~K}$ above the thermostat temperature. Once the bolometer has responded to this power input, the readout electronics is adjusted to deliver a zero voltage response in this working point condition. Then the system is locked. The carrier is a square shape $200 \mathrm{kHz}$ frequency.

Figure 6 shows typical recorded curves in the case of square power stimulation. The feedback signal rises when the input power is low and it respectively decreases when input power is high. Moreover the calculated feedback power based on a feedback voltage has the same order of magnitude as the input power. This underlies the proper functioning of the proposed method.

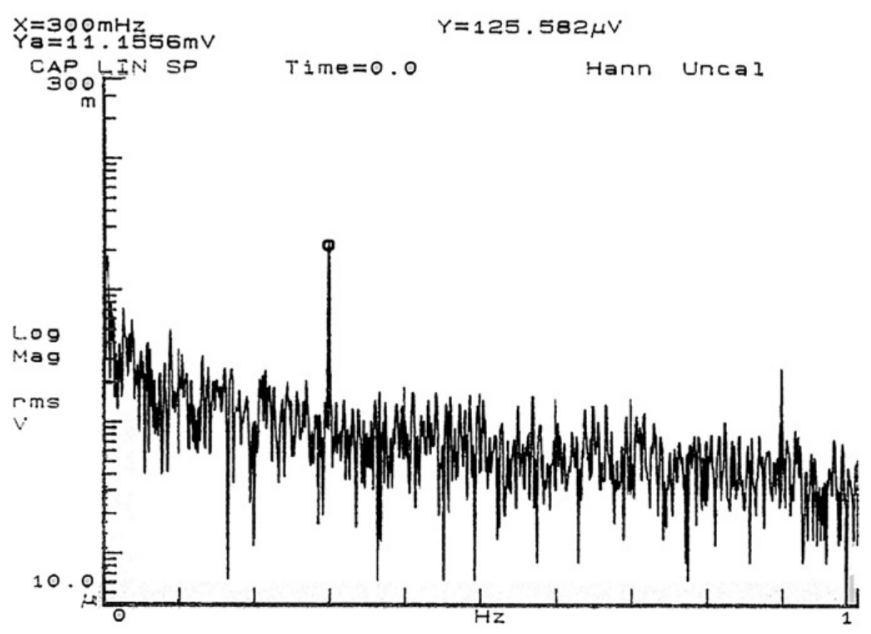

Figure 7. Open-loop output signal power spectrum in response to a $1 \mathrm{~mW}$ power stimulation modulated at $300 \mathrm{mHz}$.

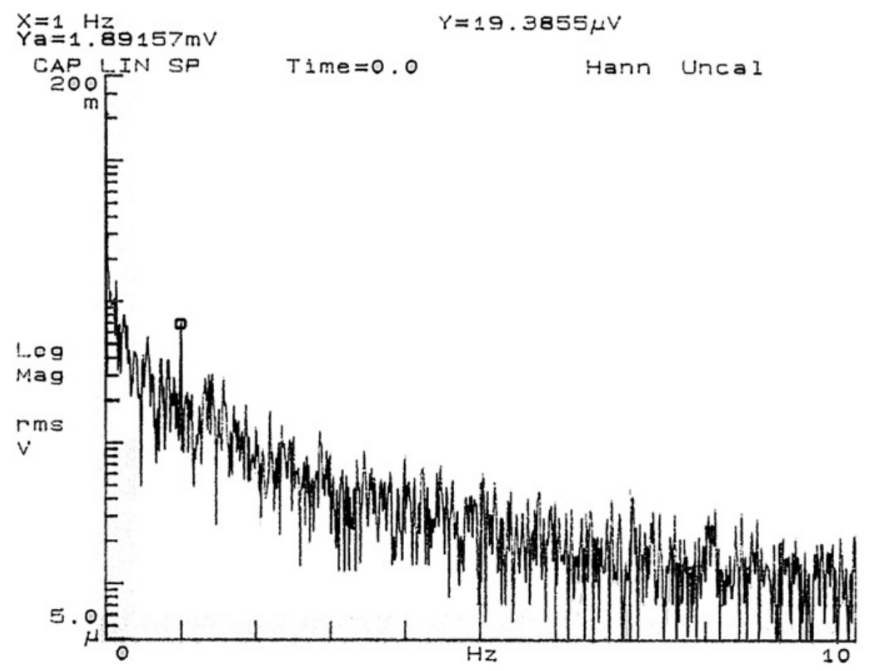

Figure 8. Open-loop output signal power spectrum in response to a $1 \mathrm{~mW}$ power stimulation modulated at $1 \mathrm{~Hz}$.

Table 1. Time constant depending on the configuration.

\begin{tabular}{llll}
\hline & Open loop & $\begin{array}{l}\text { Closed loop } \\
\text { (ES) }\end{array}$ & $\begin{array}{l}\text { Closed loop } \\
\text { (CCES) }\end{array}$ \\
\hline Time constant (s) & 11.6 & 0.9 & 0.8 \\
\hline
\end{tabular}

\section{Configuration comparison}

The same open-loop gain, $35 \mathrm{~dB}$, was achieved by properly setting the controller in both ES and CCES cases to compare these configurations on the same basis.

The time constant response was obtained through stepresponse experiments using the heating resistor $R_{\text {heating2 }}$. The power stimuli were square pulses 0 to $3.3 \mathrm{~V}$ corresponding to an applied power of $1 \mathrm{~mW}$ or 0 to $1 \mathrm{~V}$ corresponding to $100 \mu \mathrm{W}$. The results for this parameter are summarized in table 1.

A time constant reduction is checked in both closed-loop configurations. In this case, a further decrease of time constant 


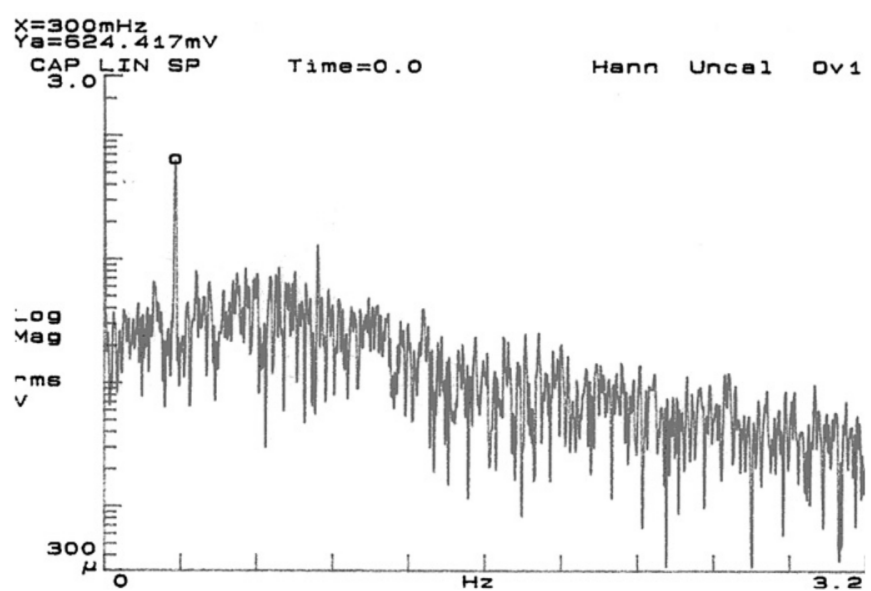

Figure 9. ES output signal power spectrum in response to a $1 \mathrm{~mW}$ power stimulation modulated at $300 \mathrm{mHz}$ of the device in the classical electrothermal substitution feedback configuration with a closed-loop gain of $35 \mathrm{~dB}$.

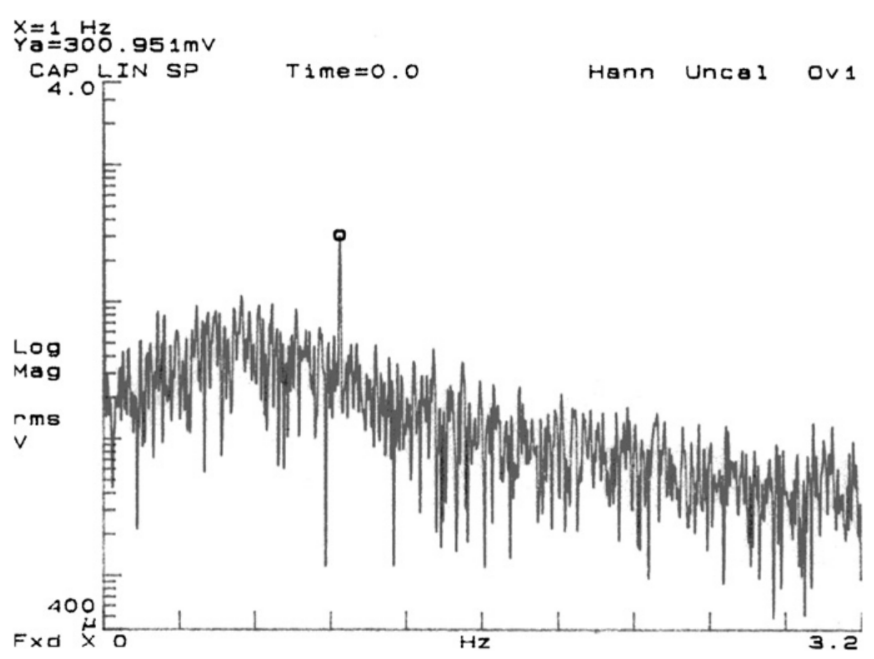

Figure 10. ES output signal power spectrum in response to a $1 \mathrm{~mW}$ power stimulation modulated at $1 \mathrm{~Hz}$ of the device in the classical electrothermal substitution feedback configuration with a closed-loop gain of $35 \mathrm{~dB}$.

was limited by the poor characteristics of the bolometer. It was so because of the bolometer's low responsivity which induced a small signal-to-noise ratio that did not allow for higher controller gains.

This signal-to-noise ratio has been studied further. Power stimulation of $1 \mathrm{~mW}$ was applied at frequencies varying between $30 \mathrm{mHz}$ and $3 \mathrm{~Hz}$. Figures 7-12 successively represent the spectral recordings in open-loop, ES closedloop and CCES closed-loop cases for two input stimulations $300 \mathrm{mHz}$ and $1 \mathrm{~Hz}$. The spectra reveal the $1 / f$ noise and the white noise of the device and its associated electronics, and the response to the input stimuli. In open loop, we observe the classical $1 / f$ noise and white noise shape due to the device and its associated electronics.

In closed loop, in contrast, a remarkable noise shape due to thermal stabilization resulting in a low frequency noise reduction is observed. The system has a high-pass profile response to noise in a closed-loop configuration that

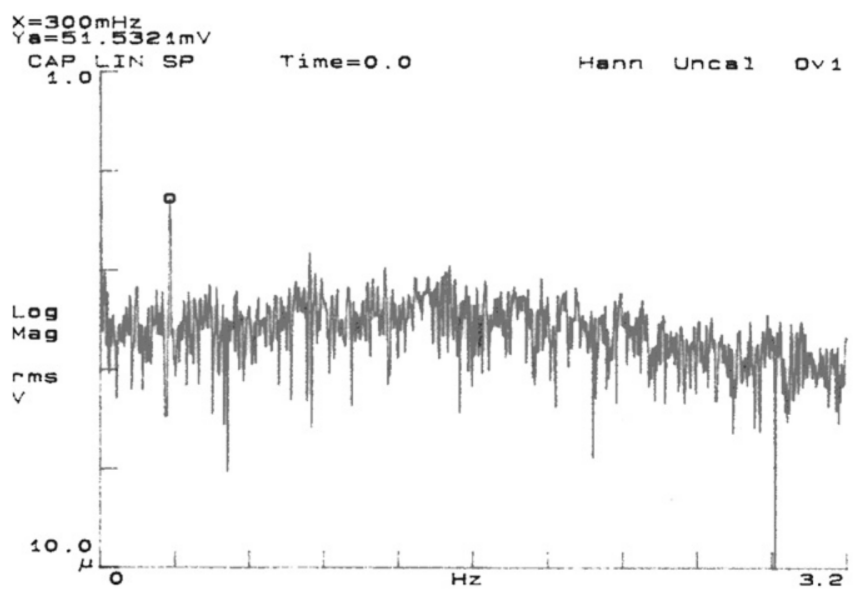

Figure 11. CCES output signal power spectrum in response to a $1 \mathrm{~mW}$ power stimulation modulated at $300 \mathrm{mHz}$ of the device in the proposed CCES feedback configuration.

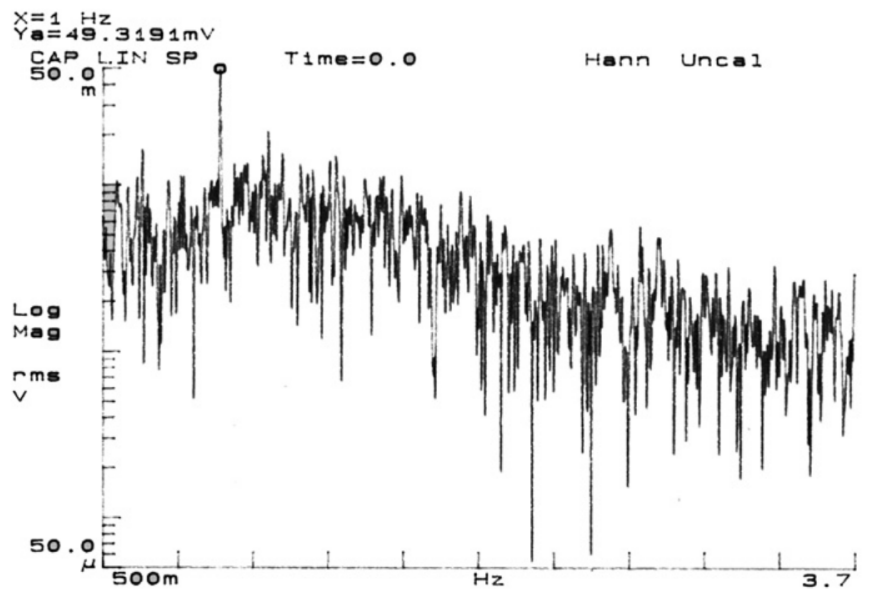

Figure 12. CCES output signal power spectrum in response to a $1 \mathrm{~mW}$ power stimulation modulated at $1 \mathrm{~Hz}$ of the device in a CCES feedback configuration.

compensates the $1 / f$ noise shape. That does not mean that the signal-to-noise ratio is improved. Indeed, the signal-tonoise ratio is still the same in open loop and closed loop as recalled in the equation hereafter. Between open- and closedloop the output measurement signal is not at the same location in each case. In open loop, the measurement signal is taken at the output of the readout electronics $\left(V\left(T_{B}\right)\right)$. In closed loop, the measurement signal is taken at the output of the controller ( $\left.V_{\text {heat feedback }}\right)$.

If we assume that the variations around the working point are small, the system equations can be linearized:

$$
V\left(T_{B}\right)_{\text {open loop }}(s)=G \cdot F(s) \cdot P_{\text {incident }}(s)+G \cdot n(s),
$$

where $n$ is the electronics first stage and device noise.

$\operatorname{SNR}_{\text {open loop }}(s)=\frac{F(s) \cdot P_{\text {incident }}(s)}{n(s)}$,

$V_{\text {heat feedback closed-loop }}(s)$

$=\frac{H_{1} \cdot V_{\mathrm{ref}}-H_{1} \cdot G \cdot F(s) \cdot n(s)-H_{1} \cdot G \cdot F(s) \cdot P_{\text {incident }}(s)}{1+H \cdot G \cdot F(s)}$, 


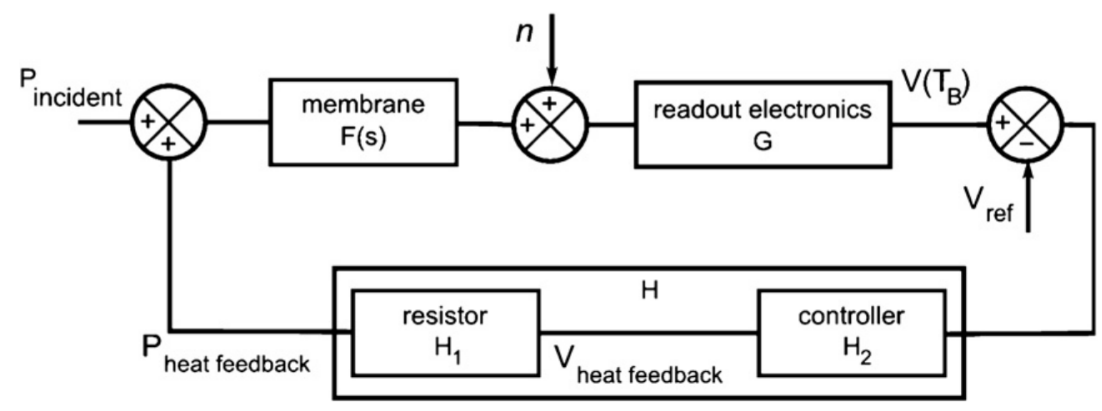

Figure 13. Block diagram of the system.

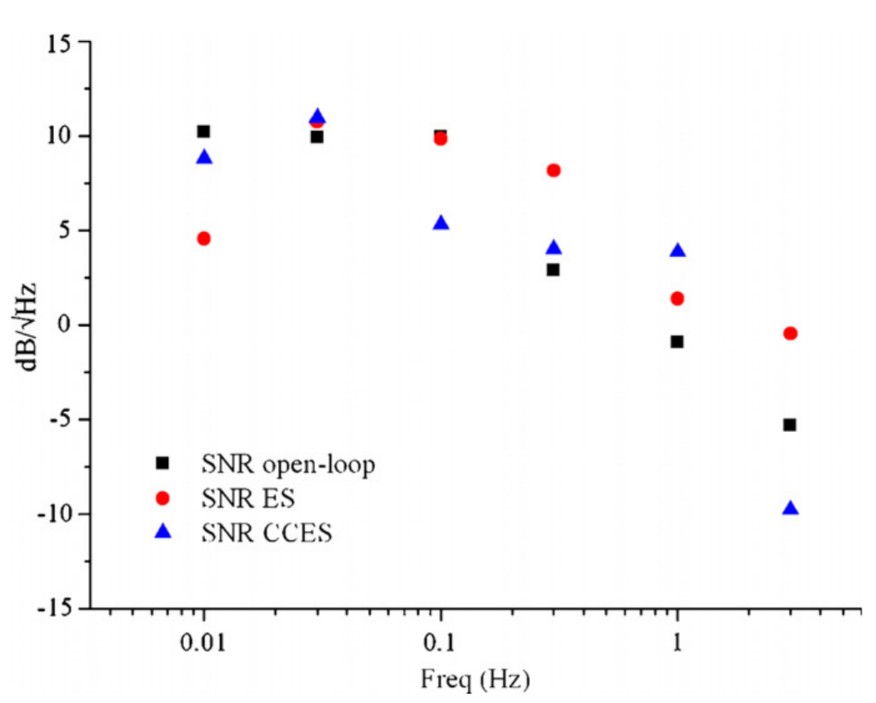

Figure 14. Signal-to-noise ratio for the three configurations.

with $H=H_{1} H_{2}$. Then, the signal-to-noise ratio is still the same:

$$
\operatorname{SNR}_{\text {closed-loop }}(s)=\frac{F(s) \cdot P_{\text {incident }}(s)}{n(s)} .
$$

The invariance of the signal-to-noise ratio is confirmed by the measures performed from $10 \mathrm{mHz}$ to $3 \mathrm{~Hz}$ as shown in figure 14. Those results stem from the spectrum obtained with the HP3562 A signal analyzer.

\section{Discussion}

Even limited by the poor responsivity of the bolometer used for the testing of the proposed method, for the first time, an electrical substitution without an added heating resistor was demonstrated. As in the case of classical electrical substitution, it allows time constant reduction and shall induce all the substantial improvements related to the implementation of a closed-loop system. But as no added heating resistor is needed to implement the CCES, it allows for simpler designs and manufacturing processes when new bolometers are concerned and can be implemented to every pre-existing bolometer.

As responsivity and time constant have opposite behavior depending on the thermal conductance, the time constant decreases due to electrical substitution feedback can be used to release the constraint on $G_{\text {th }}$ during the design of bolometers to optimize them for responsivity. Decreasing the thermal conductance will increase the responsivity while the electrical substitution configuration will allow compensation of the resulting time constant increase. Besides, saving an extra heating resistor placed next to the sensing resistor also reduces the thermal mass of the whole device, hence decreasing the time constant. This method is well suited to be combined with the current work using thin silicon or polymer membranes or micromesh for manufacturing bolometers $[7,8,18]$.

\section{Conclusion}

In this paper, a new configuration was proposed for the use of bolometers in a closed-loop electrical substitution. For the first time, the performances of the proposed configuration were evaluated on a bolometer. The performances are comparable to a standard electrical substitution feedback configuration. Since it does not require an extra power source on the bolometer, the new configuration proposed is suitable for all kinds of existing bolometers and allows new trade-offs in the design of future bolometers.

The proposed configuration can be extended to every application based on temperature measurement such as hot wire anemometry or chemical catalysis microsensors.

\section{References}

[1] Fièque $\mathrm{B}$, Tissot $\mathrm{J}$ L, Trouilleau $\mathrm{C}$, Crastes $\mathrm{A}$ and Legras $\mathrm{O}$ 2007 Uncooled microbolometer detector: recent developments at Ulis Infrared Phys. Technol. 49 187-91

[2] Richards P L 1994 Bolometers for infrared and millimeter waves J. Appl. Phys. 76 1-24

[3] Hwang T L, Schwarz S E and Rutledge D B 1979 Microbolometers for infrared detection Appl. Phys. Lett. $34773-6$

[4] Kruse P W 2001 Uncooled Thermal Imaging Arrays, Systems and Applications (Bellingham, WA: SPIE) ISBN: 0-8194-4122-8

[5] Guillet B, Méchin L, Yang F, Routoure J M, Le Dem G, Gunther C, Chakalov R A and Robbes D 2003 NET of YBCO and LSMO thermometers for bolometric applications Routoure Advanced Research Workshop (Brno, Czech Republic)

[6] Zerov V Y and Malyarov V G 2001 Heat-sensitive materials for uncooled microbolometer arrays J. Opt. Technol. 68939

[7] Atlass M 1992 Microstructure design for high IR sensitivity WO 94/01743 
[8] Mahmood A, Butler D P and Celik-Butler Z 2006 Micromachined bolometers on polyimide Sensors Actuators A 132 452-9

[9] Almasri M, Celik-Butler Z, Butler D P, Yaradanakul A and Yildiz A 2002 Uncooled multimirror broad-band infrared microbolometers J. Microelectromech. Syst. 11 528-35

[10] Danilchenko B A, Jasiukiewicz Cs, Paszkiewicz T and Wolski S 2007 Nonlinear response of superconductor bolometer to phonon fluxes Eur. Phys. J. Appl. Phys. 26 151-9

[11] Yang F, Méchin L, Routoure J-M, Guillet B and Chakalov R A 2006 Low-noise $\mathrm{La}_{0.7} \mathrm{Sr}_{0.3} \mathrm{MnO}_{3}$ thermometers for uncooled bolometric applications J. Appl. Phys. 99024903

[12] Allègre G, Guillet B, Robbes D, Méchin L, Lebargy S and Nicoletti S 2007 A room temperature $\mathrm{Si}_{3} \mathrm{~N}_{4} / \mathrm{SiO}_{2}$ membrane-type electrical substitution radiometer using thin film platinum thermometers Meas. Sci. Technol. 18 183-9

[13] Rice J P, Lorentz S R, Datla R U, Vale L R, Rudman D A, Sing M L C and Robbes D 1998 Active cavity absolute radiometer based on high- $T_{\mathrm{c}}$ superconductors Metrologia 35289

[14] Denoual M, Delaunay S, Durantel F, Guillet B, Lebargy S and Robbes D 2007 MicroBolometer on polymer membrane with heat feedback control for non radiative applications Proc. IEEE Sensors (Altanta, USA)

[15] Irwin K D 1995 An application of electrothermal feedback for high resolution cryogenic particle detection Appl. Phys. Lett. 66 1998-2000

[16] Lee A T, Gildemeister J M, Lee S F and Richards P L 1997 Voltage biased high- $T_{\mathrm{c}}$ superconducting infrared bolometers with strong electrothermal feedback IEEE Trans. Appl. Supercond. 7 2378-81

[17] Carslaw H S and Jaeger J C 1959 Conduction of Heat in Solids (Oxford: Clarendon)

[18] Turner A D, Bock J J, Beeman J W, Glenn, Hargrave P C, Hristov V V, Nguyen H T, Rahman F, Sethuraman S and Woodcraft A L 2001 Silicon nitride micromesh bolometer array for submillimeter astrophysics Appl. Opt. 404921 